\title{
Differential expression of Mart-1 in human uveal melanoma cells
}

\author{
JING WANG ${ }^{*}$, RENBING JIA*, YUTING YAO, BIYUN CUN, \\ XIAOLIN HUANG, PING GU, SHENGFANG GE and XIANQUN FAN
}

\begin{abstract}
Department of Ophthalmology, Ninth People's Hospital, Shanghai Jiaotong University, School of Medicine, Shanghai 200011, P.R. China
\end{abstract}

Received March 18, 2011; Accepted May 30, 2011

DOI: $10.3892 / \mathrm{mmr} .2011 .504$

\begin{abstract}
Uveal melanoma (UM) is one of the most common primary intraocular malignant tumors in adults. Melanoma antigen recognized by $\mathrm{T}$ cell-1 (Mart-1), one of the melanosome-specific proteins, has been widely studied as a marker recognized by cytotoxicity $\mathrm{T}$ lymphocytes. Mart-1 is considered to play a critical role in the immunotherapy for melanoma. Additionally, as a biomarker, Mart-1 is often used with other tumor-associated antigens for antidiastole in cutaneous melanoma (CM), uveal melanoma (UM) and nevus. In this study, the differential expression of Mart-1 was investigated in four human UM cells (SP6.5, VUP, OCM-1 and OM431) on three levels of analysis: messenger ribonucleic acid (mRNA), protein and, eventually, morphology. The results revealed that SP6.5 cells had high Mart-1 protein expression while VUP cells had almost none. OCM-1 and OM431 cells produced less Mart-1 than SP6.5 cells according to Western blot analysis, although OM431 cells had the highest expression of Mart-1 mRNA according to real-time PCR. The results indicate the potential use of Mart-1 in the development of therapy for UM.
\end{abstract}

\section{Introduction}

Uveal melanoma (UM) is one of the most common primary intraocular malignant tumors in adults (1). Due to drug resistance and a high likelihood of metastasis to other organs, UM has a very poor prognosis (2). In the clinic, the efficacy of conventional treatments for UM, such as chemotherapy, radiotherapy and surgical excision, is very limited. In order to overcome this obstacle, immunotherapy has been extensively

Correspondence to: Professor Xianqun Fan and Professor Shengfang Ge, Department of Ophthalmology, Ninth People's Hospital, Shanghai Jiaotong University School of Medicine, 639 Zhi Zao Ju Road, Shanghai 200011, P.R. China

E-mail: fanxq@sh163.net; geshengfang@hotmail.com

${ }^{*}$ Contributed equally

Key words: uveal melanoma, melanoma antigen recognized by $\mathrm{T}$ cell-1, differentially expressed protein studied, and has become an important component of therapy for malignant melanomas $(3,4)$.

Melanoma antigen recognized by T cell-1 (Mart-1), one of the melanocyte/melanoma differentiation antigens, is highly produced in early-stage melanosomes and is indispensable for melanosome structure and maturation in the formation of a complex with gp100 (5-7). Because it is typically characteristic of melanocytes and melanoma, Mart-1 is often used as a biomarker in combination with other tumor-associated antigens for antidiastole in cutaneous melanoma (CM), uveal melanoma (UM) and nevus, as well as melanoma micrometastases (8-10). Mart-1 has also been widely studied for many years as a marker recognized by cytotoxicity $\mathrm{T}$ lymphocytes, and plays a critical role in the immunotherapy for melanoma $(11,12)$. However, the complete mechanisms of Mart-1 activity have yet to be elucidated.

In this study, the expression of Mart-1 was examined in four human UM cell lines and one retinal pigment epithelium cell line at the messenger ribonucleic acid (mRNA), protein and morphological levels, and it was observed that these cells presented distinct properties. The results provide new information on Mart-1 that may be significant for further research and the development of treatments for UM.

\section{Materials and methods}

Cell lines and culture. The human UM cell lines, SP6.5, VUP, OCM-1 and OM431, were kindly provided by Professor John F. Marshall (Tumor Biology Laboratory, Cancer Research UK Clinical Center, John Vane Science Centre, London, UK) (13). The OCM-1 cell line was established from biopsied specimens of choroidal melanomas of spindle B cell type morphology (14). The OM431 and VUP cell lines were mainly composed of epithelioid cells, while the SP6.5 cell line was of a mixed spindle-epithelioid cell type (15). Human retinal pigmented epithelium cell ARPE-19 was generously provided by the Department of Ophthalmology, Ruijin Hospital, Shanghai Jiaotong University School of Medicine, P.R. China. The human melanoma cell line MeWo was purchased from the American Type Culture Collection (Manassas, VA, USA). The UM and MeWo cells were cultured in Dulbecco's modified Eagle's medium supplemented with $10 \%$ fetal bovine serum (FBS) at $37^{\circ} \mathrm{C}$ in a humidified incubator with $5 \% \mathrm{CO}_{2}$. ARPE-19 cells were maintained in DMEM/F12 supplemented with $10 \% \mathrm{FBS}$ at $37^{\circ} \mathrm{C}$ under $5 \% \mathrm{CO}_{2}$. 
Total RNA extraction, cDNA synthesis and reverse transcriptionpolymerase chain reaction. For reverse transcription-polymerase chain reaction (RT-PCR), total mRNA was isolated from the UM and ARPE-19 cells using TRIzol reagent (Invitrogen, Carlsbad, CA, USA) according to the manufacturer's instructions. Then, $33 \mu \mathrm{g}$ of total RNA were reverse-transcribed in a $20 \mu \mathrm{l}$ volume containing $0.5 \mathrm{mM}$ dNTPs, $2.5 \mu \mathrm{M}$ random primers, 40 units RNase inhibitor and 200 units M-MLV reverse transcriptase (Fermentas, Ontario, CA). The cDNA synthesis reaction was performed according to the manufacturer's protocol. The PCR reaction $(20 \mu \mathrm{l})$ contained $0.5 \mu \mathrm{g}$ cDNA, $0.5 \mu \mathrm{M}$ of each primer and $10 \mu \mathrm{l}$ Ex Taq solution (Takara, Tokyo, Japan). The primer sequences were as follows: Mart-1 forward, 5'-CACGGCCACTCTTACACCAC-3'; reverse, 5'-GGAGCATTGGGAACCACAGG-3'; GAPDH forward, 5'-CAAGGTCATCCATGACAACTTTG-3'; reverse, 5'-GTCCACCACCCTGTTGCTGTAG-3'. PCR cycle parameters were the same for both primers used and consisted of 30 cycles of denaturation at $94^{\circ} \mathrm{C}$ for $30 \mathrm{sec}$, annealing at $60^{\circ} \mathrm{C}$ for $40 \mathrm{sec}$ and extension at $72^{\circ} \mathrm{C}$ for $50 \mathrm{sec}$. The amplified products were analyzed by electrophoresis on $2 \%$ agarose gels, stained with ethidium bromide and visualized under UV light.

Quantitative real-time PCR. Using a previously synthesized cDNA template and method, SYBR quantitative real-time PCR was performed (16). The samples were examined using an ABI PRISM 7900 HT sequence detector (Applied Biosystems, Foster City, CA, USA) according to the manufacturer's instructions (Takara), and the data were normalized against the CT (threshold cycle) of the GAPDH control.

Western blot analysis. Mart-1 protein expression in the UM and ARPE-19 cells was examined by Western blot analysis as previously described (17). The cells were harvested, washed in cold phosphate-buffered saline (PBS) and lysed with lysis buffer (Fermentas). The protein samples were then equally divided using the BCA protein assay kit (Thermo, Rockford, IL, USA). Equal amounts of protein were analyzed using the Bio-Rad protein assay (Bio-Rad Laboratories, Hercules, CA, USA) separated by sodium dodecyl sulfate-polyacrylamide gel electrophoresis on $12 \%$ (wt/vol) polyacrylamide gels, then transferred to polyvinylidene fluoride membranes. These were subsequently incubated with a secondary antibody conjugated to a fluorescent tag. Finally, the bands were visualized using the Odyssey Infrared Imaging System (LI-COR, Lincoln, NE, USA). The antibodies used were M2-7C10 (Abcam, Hong Kong SAR, P.R. China) and anti- $\beta$-actin (Sigma, St. Louis, MO, USA).

Immunocytochemical analysis. Cells were collected and attached to glass slides, then these cell smears were fixed by 4\% paraformaldehyde for $30 \mathrm{~min}$ and incubated with $0.1 \%$ Triton X-100, 5\% dimethylsulfoxide in PBS for $10 \mathrm{~min}$. The cells were subsequently blocked with normal goat serum (Invitrogen) at $37^{\circ} \mathrm{C}$ for $30 \mathrm{~min}$. After rinsing with cold PBS twice, the cells were incubated with M2-7C10 antibody against Mart- 1 at $4^{\circ} \mathrm{C}$ overnight. After another wash in PBS, the cells were stained by DAB (Sunbio, Shanghai, P.R. China).
$\mathbf{A}$

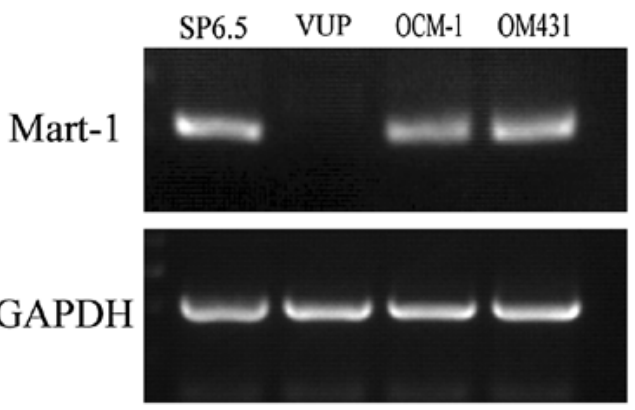

B

Mart-1 mRNA Expression

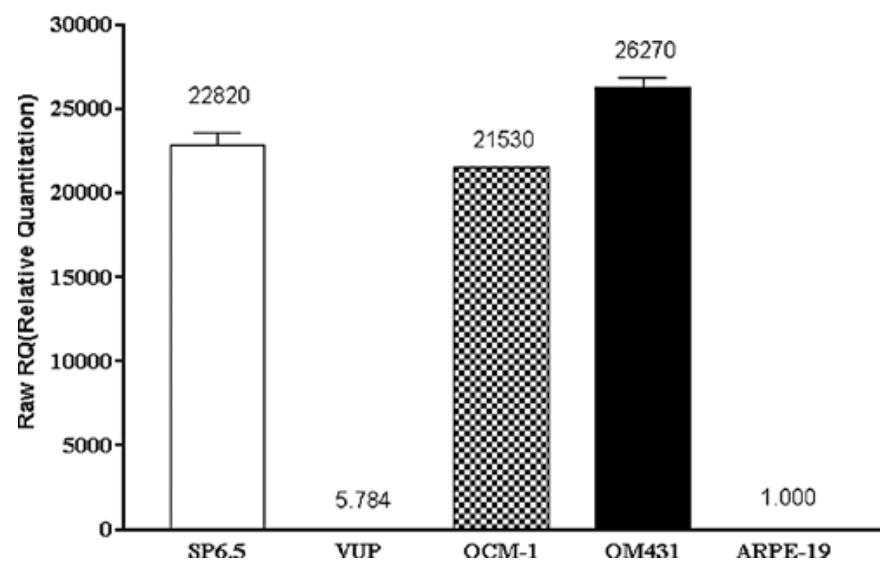

Figure 1. Human UM cells express Mart-1 mRNA. Mart-1 mRNA expressions in the 4 UM cell lines were tested by RT-PCR. (A-B) The relative quantitative analysis of Mart-1 mRNA expression in the 4 UM cells and ARPE-19 cells was determined by quantitative real-time PCR. Bars represent the means \pm SD of 3 independent experiments, and GAPDH was tested as the endo-control.

Nuclear counterstaining was performed with hematoxylin for 2 min.

Immunofluorescence staining. Cells were prepared as described above, but were incubated with the mouse monoclonal antibody M2-7C10 at $37^{\circ} \mathrm{C}$ for only $1 \mathrm{~h}$. Subsequently, the cells were gently rinsed twice in cold PBS, then incubated with goat anti-mouse $\operatorname{IgG}$ secondary antibody (1:1000 dilution in PBS/0.5\% BSA; Invitrogen) and DAPI (4',6-diamidino2-phenylindole, $1: 1000$ dilution in $\mathrm{PBS} / 0.5 \% \mathrm{BSA}$ ) at $37^{\circ} \mathrm{C}$ for $30 \mathrm{~min}$ in the dark. The cell smears were finally placed on coverslips and photographed under a fluorescence microscope at $490-520 \mathrm{~nm}$.

\section{Results}

Expression of Mart-1 in UM cells at the mRNA level. Using the human retinal pigment epithelium cell line, ARPE-19, as the control, we first investigated the mRNA expression of Mart-1 in four human UM cell lines (SP6.5, VUP, OCM-1 and OM431). The results of PR-PCR clearly showed that the control ARPE-19 cells did not express Mart-1, while the UM cells expressed Mart-1 mRNA, with the exception of VUP cells (Fig. 1A). To compare differences in Mart-1 mRNA expression between these cell lines, real-time PCR 
A

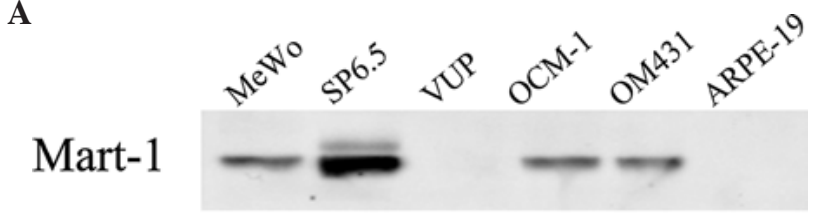

\section{$\beta$-actin}

B

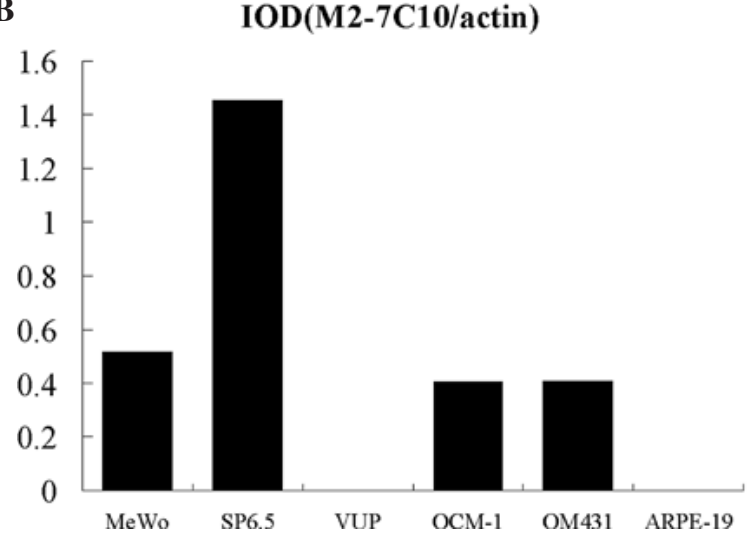

Figure 2. Human UM cells express Mart-1 protein. Western blot analysis for the protein level of Mart-1 expression was performed using the antibody, M2-7C10, in these 6 melanocytes. (A) The immune detection of $\beta$-actin was used as a loading control. MeWo was used as the Mart-1-positive control while ARPE-19 was the negative control. (B) The protein bands were analyzed by gradation scan and the relative value M2-7C10/ $\beta$-actin.

was performed. The results revealed that the OM431 cells expressed the highest level of Mart-1 mRNA when the expression of the ARPE-19 cells was taken as 1 (Fig. 1B). Mart-1 gene expression in the OCM-1 and SP6.5 cells was detected at similarly low levels compared to the OM431 cells. Consistent with the results of RT-PCR, the VUP cells expressed negligible levels of Mart-1.
M2-7C10 Blank

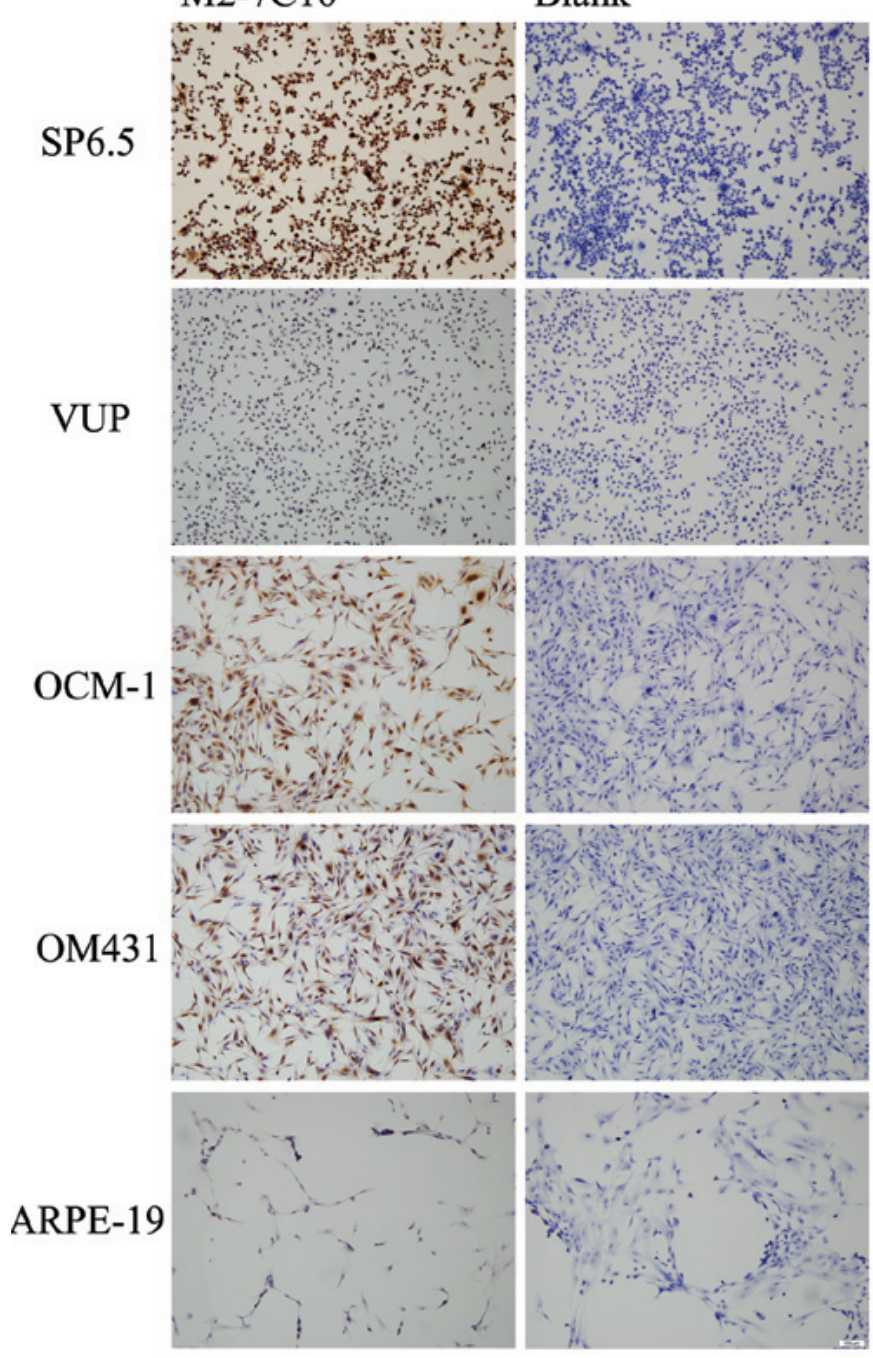

Figure 3. Immunohistochemical staining of Mart-1 in 4 UM cells and ARPE-19 cells. Nuclei were counterstained with hematoxylin (blue), and Mart-1 was visualized with diaminobenzidine (brown). The blank group was performed using PBS instead of M2-7C10. Original magnification, x10. Scale was the length of $100 \mu \mathrm{m}$.
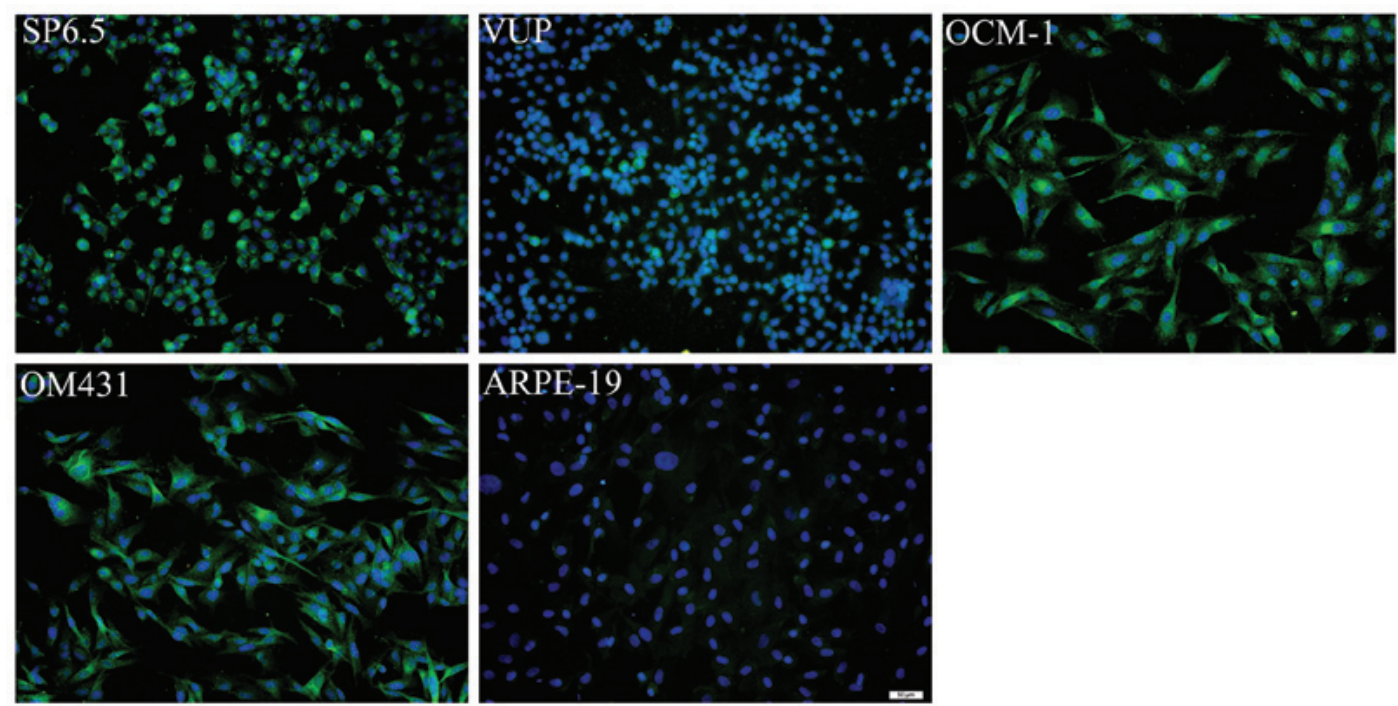

Figure 4. Immunofluorescence detection of Mart-1 in 4 UM cells and ARPE-19 cells. Nuclei were stained with DAPI (blue), and Mart-1 was visualized with goat anti-mouse IgG secondary antibody (green). Original magnification, x20. Scale was the length of $50 \mu \mathrm{m}$. 
Expression of Mart-1 in UM cells at the protein level. As Mart-1 positive controls, we used the melanoma cell line MeWo. Mart-1 protein expression in UM and ARPE-19 cells was examined by Western blot analysis. As shown in Fig. 2, Mart-1 was not present in either the APRE-19 or the VUP cells. Otherwise, Sp6.5 cells strongly expressed Mart-1 while OCM-1 and OM431 cells had relatively lower protein levels, which were the same as in MeWo cells.

Expression of Mart-1 in UM cells at the cellular level. It has been reported that Mart-1 localizes in the endoplasmic reticulum, the trans-Golgi network and the melanosome (18). We used immunocytochemical analysis to examine the expression of Mart-1 in these cells. Compared to the control group, it was clear that SP6.5 cells were markedly stained (Fig. 3), while both Arpe-19 and VUP cells had almost no staining. In addition, OCM-1 and OM431 cells were positively stained. Immunofluorescence staining confirmed the presence of Mart-1 protein expression in these cells (Fig. 4).

\section{Discussion}

Two of the major obstacles encountered in the treatment of UM are drug resistance and a high rate of metastasis. With the development of gene research, the significance and utility of gene therapy and immunotherapy have increased. A series of proteins have been extensively studied in UM, including Mart-1.

Mart-1 is a type III transmembrane protein that localizes in the endoplasmic reticulum, the trans-Golgi network and the melanosome in most melanocyte and melanoma cells. It has been reported that Mart-1 forms a complex with gp100 in an early subcellular compartment, which affects the expression, stability and processing of gp100 at the post-transcriptional level (7). This suggests that Mart-1 plays a crucial role in maintaining the normal function of melanogenesis.

Mart-1 was first identified and named by Kawakami et al in 1994 due to the fact that it could be widely recognized by autologous and allogenic tumor-infiltrating lymphocytes in human melanoma (19). Mart-1 induced lymphocytes to generate cytotoxic $\mathrm{T}$ lymphocytes in the peripheral blood of melanoma patients resulting in an effect against tumor tissue (20). As a biomarker, the specific antigen, Mart-1, could also be recognized by monoclonal antibodies M2-7C10, and exhibited a high sensitivity and specificity in primary melanoma and metastatic melanoma (21). Moreover, it has been reported that the presence of Mart-1 transcripts is a prognostic factor for subsequent development of metastases in patients with high-risk primary UM (22). Recently, vaccines generated by Mart-1 have been widely studied and the feasibility of inducing specific $\mathrm{T}$ cell responses has been revealed $(23,24)$. Although significant effort has been made, there are still many remaining questions regarding Mart-1.

Our study shows that the expression of Mart-1 is markedly different in four UM cells. This indicates that the effects of immunotherapy mediated by the antigenicity of Mart-1 among patients with UM can differ substantially, which clearly leads to distinctly varied prognoses. Furthermore, we observed that mRNA expression of Mart-1 in OCM-1 and SP6.5 cells occurred at similar levels which were slightly lower than in the OM431 cells, while the Mart-1 protein level in SP6.5 cells was much higher than in the OCM-1 and OM431 cells. The presence of two different levels indicates that there are other unknown factors, such as functional elements or proteins, regulating the translation of the Mart-1 protein at the posttranscriptional level. These uncertain factors could be the key to the immunotherapy for melanoma. Clearly, our research is just beginning to develop our understanding of Mart-1 expression in UM cells. Notably, the functional significance and regulatory mechanism of Mart-1 have yet to be elucidated.

In conclusion, our results indicate that Mart-1 is differently expressed in four UM cells (SP6.5, VUP, OCM-1 and OM431). Mart-1 mRNA was at a higher level in the OM431 cells than the OCM-1 and SP6.5 cells, while SP6.5 cells had the maximum Mart-1 protein level. However, ARPE-19 and VUP cells had no Mart-1 mRNA and protein. This could provide new insights into the characteristics of Mart-1 expression in UM cells and could lead to the development of immunotherapy for UM.

\section{Acknowledgements}

This study was supported by grants from the Shanghai Leading Academic Discipline Project (no. S30205), the National Key Program for Basic Research of China (no. 2010CB529902), the National Natural Science Foundation of China (nos. 30901654 and 81001008) and the Science and Technology Committee of Shanghai (nos. 10JC1409100 and 08410702300).

\section{References}

1. Bedikjan AY: Metastatic uveal melanoma therapy: current options. Int Ophthalmol Clin 46: 151-166, 2006.

2. Flaherty LE, Unger JM, Liu PY, Mertens WC and Sondak VK: Metastatic melanoma from intraocular primary tumors: the Southwest Oncology Group experience in phase II advanced melanoma clinical trials. Am J Clin Oncol 21: 568-572, 1998.

3. Alexandrescu DT, Ichim TE, Riordan NH, Marincola FM, Di Nardo A, Kabigting FD and Dasanu CA: Immunotherapy for melanoma: current status and perspectives. J Immunother 33: 570-590, 2010.

4. Jandus C, Speiser D and Romero P: Recent advances and hurdles in melanoma immunotherapy. Pigment Cell Melanoma Res 22: 711-723, 2009.

5. Kawakami Y, Battles JK, Kobayashi T, et al: Production of recombinant MART-1 proteins and specific antiMART-1 polyclonal and monoclonal antibodies: use in the characterization of the human melanoma antigen MART-1. J Immunol Meth 202: 13-25, 1997.

6. Kushimoto T, Basrur V, Matsunaga J, Vieira WD, Muller J, Appella $\mathrm{E}$ and Hearing VJ: A model for melanosome biogenesis based on the purification and analysis of early melanosomes. Proc Natl Acad Sci USA 98: 10698-10703, 2001.

7. Hoashi T, Watabe H, Muller J, Yamaguchi Y, Vieira WD and Hearing VJ: MART-1 is required for the function of the melanosomal matrix protein PMEL17/GP100 and the maturation of melanosomes. J Biol Chem 280: 14006-14016, 2005.

8. De Vries TJ, Trancikova D, Ruiter DJ and Van Muijen GN: High expression of immunotherapy candidate proteins gp100, MART-1, tyrosinase and TRP-1 in uveal melanoma. Br J Cancer 78: 1156-1161, 1998.

9. Shidham VB, Qi D, Rao RN, et al: Improved immunohistochemical evaluation of micrometastases in sentinel lymph nodes of cutaneous melanoma with 'MCW melanoma cocktail'-a mixture of monoclonal antibodies to MART-1, Melan-A, and tyrosinase. BMC Cancer 3: 15, 2003.

10. Heegaard S, Jensen OA and Prause JU: Immunohistochemical diagnosis of malignant melanoma of the conjunctiva and uvea: comparison of the novel antibody against melan-A with S100 protein and HMB-45. Melanoma Res 10: 350-354, 2000. 
11. Kawakami Y, Robbins PF, Wang RF, Parkhurst MR, Kang X and Rosenberg SA: The use of melanosomal proteins in the immunotherapy of melanoma. J Immunother 21: 237-246, 1998.

12. Zippelius A, Pittet MJ, Batard P, et al: Thymic selection generates a large $\mathrm{T}$ cell pool recognizing a self-peptide in humans. J Exp Med 195: 485-494, 2002.

13. Jia R, Jiao Z, Xu X, et al: Functional significance of B7-H1 expressed by human uveal melanoma cells. Mol Med Rep 4 163-167, 2011.

14. Kan-Mitchell J, Mitchell MS, Rao $N$ and Liggett PE: Characterization of uveal melanoma cell Lines that grow as xenografts in rabbit eyes. Invest Ophthalmol Vis Sci 30 829-834, 1989

15. Albert DM, Ruzzo MA, McLaughlin MA, Robinson NL, Craft JL and Epstein J: Establishment of cell lines of uveal melanoma. Invest Ophthalmol Vis Sci 25: 1284-1299, 1984.

16. Zhou Y, Song X, Jia R, et al: Radiation-inducible human tumor necrosis factor-related apoptosis-inducing ligand (TRAIL) gene therapy: a novel treatment for radioresistant uveal melanoma. Pigment Cell Melanoma Res 23: 661-674, 2010.

17. Song X, Zhou Y, Jia R, et al: Inhibition of retinoblastoma in vitro and in vivo with conditionally replicating oncolytic adenovirus H101. Invest Ophthalmol Vis Sci 51: 2626-2635, 2010

18. Rimoldi D, Muehlethaler K, Salvi S, Valmori D, Romero P, Cerottini JC and Levy F: Subcellular localization of the melanoma-associated protein Melan-A (MART-1) influences the processing of its HLA-A2-restricted epitope. J Biol Chem 276: 43189-43196, 2001.
19. Kawakami Y, Eliyahu S, Delgado $\mathrm{CH}$, et al: Cloning of the gene coding for a shared human melanoma antigen recognized by autologous $\mathrm{T}$ cells infiltrating into tumor. Proc Natl Acad Sci USA 91: 3515-3519, 1994.

20. Stevens EJ, Jacknin L, Robbins PF, Kawakami Y, El-Gamil M, Rosenberg SA and Yannelli JR: Generation of tumor-specific CTLs from melanoma patients by using peripheral blood stimulated with allogeneic melanoma tumor cell lines. Fine specificity and MART-1 melanoma antigen recognition. J Immunol 154: 762-771, 1995.

21. Ohsie SJ, Sarantopoulos GP, Cochran AJ and Binder SW: Immunohistochemical characteristics of melanoma. J Cutan Pathol 35: 433-444, 2008

22. Schuster R, Bechrakis NE, Stroux A, et al: Circulating tumor cells as prognostic factor for distant metastases and survival in patients with primary uveal melanoma. Clin Cancer Res 13: 1171-1178, 2007.

23. Lienard D, Avril MF, Le Gal FA, et al: Vaccination of melanoma patients with Melan-A/Mart-1 peptide and Klebsiella outer membrane protein $\mathrm{p} 40$ as an adjuvant. J Immunother 32: 875-883, 2009.

24. Riemann H, Takao J, Shellman YG, et al: Generation of a prophylactic melanoma vaccine using whole recombinant yeast expressing MART-1. Exp Dermatol 16: 814-822, 2007. 